\title{
Article
}

\section{Congruence properties of indices of triangular numbers multiple of other triangular numbers}

\author{
Vladimir Pletser \\ European Space Agency (ret.); pletservladimir@gmail.com \\ Academic Editor: Tuncer Acar \\ Received: 5 March 2021; Accepted: 10 June 2021; Published: 14 July 2021.
}

\begin{abstract}
For any non-square integer multiplier $k$, there is an infinity of triangular numbers multiple of other triangular numbers. We analyze the congruence properties of indices $\xi$ of triangular numbers multiple of triangular numbers. Remainders in congruence relations $\xi$ modulo $k$ come always in pairs whose sum always equal $(k-1)$, always include 0 and $(k-1)$, and only 0 and $(k-1)$ if $k$ is prime, or an odd power of a prime, or an even square plus one or an odd square minus one or minus two. If the multiplier $k$ is twice the triangular number of $n$, the set of remainders includes also $n$ and $\left(n^{2}-1\right)$ and if $k$ has integer factors, the set of remainders include multiples of a factor following certain rules. Algebraic expressions are found for remainders in function of $k$ and its factors, with several exceptions. This approach eliminates those $\xi$ values not providing solutions.
\end{abstract}

Keywords: Triangular numbers; Multiple of triangular numbers; Recurrent relations; Congruence properties.

MSC: Primary 11A25; Secondary 11D09.

\section{Introduction}

$\mathbf{T}$

riangular numbers $T_{t}=\frac{t(t+1)}{2}$ are one of the figurate numbers enjoying many properties; see, e.g., [1,2] for relations and formulas. Triangular numbers $T_{\xi}$ that are multiples of other triangular number $T_{t}$

$$
T_{\xi}=k T_{t}
$$

are investigated. Only solutions for $k>1$ are considered as the cases $k=0$ and $k=1$ yield respectively $\xi=0$ and $\xi=t, \forall t$. Accounts of previous attempts to characterize these triangular numbers multiple of other triangular numbers can be found in [3-9]. Recently, Pletser [9] showed that, for non-square integer values of $k$, there are infinitely many solutions that can be represented simply by recurrent relations of the four variables $t, \xi$, Tt and $T_{\xi}$, involving a rank $r$ and parameters $\kappa$ and $\gamma$, which are respectively the sum and the product of the $(r-1)^{\text {th }}$ and the $r^{\text {th }}$ values of $t$. The rank $r$ is being defined as the number of successive values of $t$ solutions of (1) such that their successive ratios are slowly decreasing without jumps.

In this paper, we present a method based on the congruent properties of $\xi(\bmod k)$, searching for expressions of the remainders in function of $k$ or its factors. This approach accelerates the numerical search of the values of $t_{n}$ and $\xi_{n}$ that solve (1), as it eliminates values of $\xi$ that are known not to provide solutions to (1). The gain is typically in the order of $k / v$ where $v$ is the number of remainders, which is usually such that $v \ll k$.

\section{Rank and Recurrent Equations}

Sequences of solutions of (1) are known for $k=2,3,5,6,7,8,10$ and are listed in the Online Encyclopedia of Integer Sequences (OEIS) [10], with references given in Table 1.

Among all solutions, $t=0$ is always a first solution of (1) for all non-square integer value of $k$, yielding $\xi=0$.

Let's consider the two cases of $k=2$ and $k=7$ yielding the successive solution pairs as shown in Table 2. We indicate also the ratios $t_{n} / t_{n-1}$ for both cases and $t_{n} / t_{n-2}$ for $k=7$. It is seen that for $k=2$, the ratio $t_{n} / t_{n-1}$ varies between close values, from 7 down to 5.829 , while for $k=7$, the ratio $t_{n} / t_{n-1}$ alternates between values $2.5 \quad \ldots 2.216$ and $7.8 \ldots 7.23$, while the ratio $t_{n} / t_{n-2}$ decreases regularly from 19.5 to 16.023 
Table 1. OEIS [10] references of sequences of integer solutions of (1) for $k=2,3,5,6,7,8,10$

\begin{tabular}{c|c|c|c|c|c|c|c}
$k$ & 2 & 3 & 5 & 6 & 7 & 8 & 10 \\
\hline$t$ & $\mathrm{~A} 053141$ & $\mathrm{~A} 061278$ & $\mathrm{~A} 077259$ & $\mathrm{~A} 077288$ & $\mathrm{~A} 077398$ & $\mathrm{~A} 336623$ & $\mathrm{~A} 341893$ \\
\hline$\xi$ & $\mathrm{A} 001652$ & $\mathrm{~A} 001571$ & $\mathrm{~A} 077262$ & $\mathrm{~A} 077291$ & $\mathrm{~A} 077401$ & $\mathrm{~A} 336625$ & $\mathrm{~A} 341895$ \\
\hline$T_{t}$ & $\mathrm{~A} 075528$ & $\mathrm{~A} 076139$ & $\mathrm{~A} 077260$ & $\mathrm{~A} 077289$ & $\mathrm{~A} 077399$ & $\mathrm{~A} 336624$ & $\mathrm{~A} 068085$ \\
\hline$T_{\zeta}$ & $\mathrm{A} 029549$ & $\mathrm{~A} 076140$ & $\mathrm{~A} 077261$ & $\mathrm{~A} 077290$ & $\mathrm{~A} 077400$ & $\mathrm{~A} 336626$ & -
\end{tabular}

Table 2. Solutions of (1) for $k=2,7$

\begin{tabular}{l|r|r|l|r|r|l|l}
\multirow{2}{*}{$n$} & \multicolumn{3}{|c|}{$k=2$} & \multicolumn{5}{|c}{$k=7$} \\
\cline { 2 - 8 } & $t_{n}$ & $\xi_{n}$ & $\frac{t_{n}}{t_{n-1}}$ & $t_{n}$ & $\xi_{n}$ & $\frac{t_{n}}{t_{n-1}}$ & $\frac{t_{n}}{t_{n-2}}$ \\
\hline 0 & 0 & 0 & & 0 & 0 & & \\
\hline 1 & 2 & 3 & - & 2 & 6 & - & - \\
\hline 2 & 14 & 20 & 7 & 5 & 14 & 2.5 & - \\
\hline 3 & 84 & 119 & 6 & 39 & 104 & 7.8 & 19.5 \\
\hline 4 & 492 & 696 & 5.857 & 87 & 231 & 2.231 & 17.4 \\
\hline 5 & 2870 & 4059 & 5.833 & 629 & 1665 & 7.230 & 16.128 \\
\hline 6 & 16730 & 23660 & 5.829 & 1394 & 3689 & 2.216 & 16.023
\end{tabular}

(corresponding approximately to the product of the alternating values of the ratio $t_{n} / t_{n-1}$ ). We call rank $r$ the integer value such that $t_{n} / t_{n-r}$ is approximately constant or, better, decreases regularly without jumps (a more precise definition is given further). So, here, the case $k=2$ has rank $r=1$ and the case $k=7$ has rank $r=2$.

In [9], we showed that the rank $r$ is the index of $t_{r}$ and $\xi_{r}$ solutions of (1) such that

$$
\kappa=t_{r}+t_{r-1}=\xi_{r}-\xi_{r-1}-1,
$$

and that the ratio $t_{2 r} / t_{r}$, corrected by the ratio $t_{r-1} / t_{r}$, is equal to a constant $2 \kappa+3$

$$
\frac{t_{2 r}-t_{r-1}}{t_{r}}=2 \kappa+3
$$

For example, for $k=7$ and $r=2$, (2) and (3) yield respectively, $\kappa=7$ and $2 \kappa+3=17$.

Four recurrent equations for $t_{n}, \xi_{n}, T_{t_{n}}$ and $T_{\xi_{n}}$ are given in [9] for each non-square integer value of $k$

$$
\begin{aligned}
t_{n} & =2(\kappa+1) t_{n-r}-t_{n-2 r}+\kappa, \\
\xi_{n} & =2(\kappa+1) \xi_{n-r}-\xi_{n-2 r}+\kappa, \\
T_{t_{n}} & =\left(4(\kappa+1)^{2}-2\right) T_{t_{n-r}}-T_{t_{n-2 r}}+\left(T_{\kappa}-\gamma\right), \\
T_{\xi_{n}} & =\left(4(\kappa+1)^{2}-2\right) T_{\xi_{n-r}}-T_{\xi_{n-2 r}}+k\left(T_{\kappa}-\gamma\right),
\end{aligned}
$$

where coefficients are functions of two constants $\kappa$ and $\gamma$, respectively the sum $\kappa$ and the product $\gamma=t_{r-1} t_{r}$ of the first two sequential values of $t_{r}$ and $t_{r-1}$. Note that the first three relations (4) to (6) are independent of the value of $k$.

\section{Congruence of $\xi$ modulo $k$}

We use the following notations: for $A, B, C \in \mathbb{Z}, B<C, C>1, A \equiv B(\bmod C)$ means that $\exists D \in \mathbb{Z}$ such that $A=D C+B$, where $B$ and $C$ are called respectively the remainder and the modulus. To search numerically for the values of $t_{n}$ and $\xi_{n}$ that solve (1), one can use the congruent properties of $\xi(\bmod k)$ given in the following propositions. In other words, we search in the following propositions for expressions of the remainders in function of $k$ or its factors.

Proposition 1. For $\forall s, k \in \mathbb{Z}^{+}, k$ non-square, $\exists \xi, \mu, v, i, j \in \mathbb{Z}^{+}$, such that if $\xi_{i}$ are solutions of (1), then for $\xi_{i} \equiv$ $\mu_{j}(\bmod k)$ with $1 \leq j \leq v$, the number $v$ of remainders is always even, $v \equiv 0(\bmod 2)$, the remainders come in pairs 
whose sum is always equal to $(k-1)$, and the sum of all remainders is always equal to the product of $(k-1)$ and the number of remainder pairs, $\sum_{j=1}^{v} \mu_{j}=(k-1) v / 2$.

Proof. Let $s, i, j, k, \xi, \mu, v, \alpha, \beta \in \mathbb{Z}^{+}, k$ non-square, and $\xi_{i}$ solutions of (1). Rewriting (1) as $T_{t_{i}}=T_{\widetilde{\xi}_{i}} / k$, for $T_{t_{i}}$ to be integer, $k$ must divide exactly $T_{\xi_{i}}=\xi_{i}\left(\xi_{i}+1\right) / 2$, i.e., among all possibilities, $k$ divides either $\xi_{i}$ or $\left(\xi_{i}+1\right)$, yielding two possible solutions $\xi_{i} \equiv 0(\bmod k)$ or $\xi_{i} \equiv-1(\bmod k)$, i.e., $v=2$ and the set of $\mu_{j}$ includes $\{0,(k-1)\}$. This means that $\xi_{i}$ are always congruent to either 0 or $(k-1)$ modulo $k$ for all non-square values of $k$.

Furthermore, if some $\xi_{i}$ are congruent to $\alpha$ modulo $k$, then other $\xi_{i}$ are also congruent to $\beta$ modulo $k$ with $\beta=(k-\alpha-1)$. As $\xi_{i} \equiv \alpha(\bmod k)$, then $\xi_{i}\left(\xi_{i}+1\right) / 2 \equiv(\alpha(\alpha+1) / 2)(\bmod k)$ and replacing $\alpha$ by $\alpha=(k-\beta-1)$ yields $(\alpha(\alpha+1) / 2)=((k-\beta-1)(k-\beta) / 2)$, giving $\xi_{i}\left(\xi_{i}+1\right) / 2 \equiv$ $((k-\beta-1)(k-\beta) / 2)(\bmod k) \equiv(\beta(\beta+1) / 2)(\bmod k)$. In this case, $v=4$ and the set of $\mu_{j}$ includes, but not necessarily limits to, $\{0, \alpha,(k-\alpha-1),(k-1)\}$.

Note that in some cases, $v>4$, as for $k=66,70,78,105, \ldots, v=8$. However, in some other cases, $v=2$ only and the set of $\mu_{j}$ contains only $\{0,(k-1)\}$, as shown in the next proposition. In this proposition, several rules $(\mathrm{R})$ are given constraining the congruence characteristics of $\xi_{i}$.

Proposition 2. For $\forall s, k, \alpha, n \in \mathbb{Z}^{+}, k$ non-square, $\alpha>1, \exists \xi, \mu, v, i \in \mathbb{Z}^{+}$, such that if $\xi_{i}$ are solutions of (1), then $\xi_{i}$ are always only congruent to 0 and $(k-1)$ modulo $k$, and $v=2$ if either

(R1) $k$ is prime, or

(R2) $k=\alpha^{n}$ with $\alpha$ prime and $n$ odd, or

(R3) $k=s^{2}+1$ with s even, or

(R4) $k=s^{\prime 2}-1$ or (R5) $k=s^{\prime 2}-2$ with $s^{\prime}$ odd.

Proof. Let $s, s^{\prime}, k, \alpha>1, n, i, \xi, \mu, v \in \mathbb{Z}^{+}, k$ non-square, and $\xi_{i}$ are solutions of (1).

(R1)+(R2): If $k$ is prime or if $k=\alpha^{n}$ (with $\alpha$ prime and $n$ odd as $k$ is non-square), then, in both cases, $k$ can only divide either $\xi_{i}$ or $\left(\xi_{i}+1\right)$, yielding the two congruences $\xi_{i} \equiv 0(\bmod k)$ and $\xi_{i} \equiv-1(\bmod k)$.

(R3): If $k=s^{2}+1$ with $s$ even, the rank $r$ is always $r=2$ [11], and the only two sets of solutions are

$$
\begin{aligned}
& \left(t_{1}, \xi_{1}\right)=\left(s(s-1),\left(s^{2}+1\right)(s-1)\right) \\
& \left(t_{2}, \xi_{2}\right)=\left(s(s+1),\left(s^{2}+1\right)(s+1)-1\right)
\end{aligned}
$$

as can be easily shown. For $t_{1}$, forming

$$
\begin{aligned}
k T_{t_{1}} & =\frac{1}{2}\left(s^{2}+1\right)(s(s-1))(s(s-1)+1) \\
& =\frac{1}{2}\left[\left(s^{2}+1\right)(s-1)\right]\left[\left(s^{2}+1\right)(s-1)+1\right]=T_{\xi_{1}}
\end{aligned}
$$

which is the triangular number of $\xi_{1}$. One obtains similarly $\xi_{2}$ from $t_{2}$. These two relations (8) and (9) show respectively that $\xi_{1}$ is congruent to 0 modulo $k$ and $\xi_{2}$ is congruent to $(k-1)$ modulo $k$.

(R4): For $k=s^{\prime 2}-1$ with $s^{\prime}$ odd, the rank $r=2$ [11], and the only two sets of solutions are

$$
\begin{aligned}
& \left(t_{1}, \xi_{1}\right)=\left(\left(s^{\prime}-1\right) s^{\prime}-1,\left(s^{2}-1\right)\left(s^{\prime}-1\right)-1\right) \\
& \left(t_{2}, \xi_{2}\right)=\left(\left(s^{\prime}-1\right)\left(s^{\prime}+2\right)+1,\left(s^{\prime 2}-1\right)\left(s^{\prime}+1\right)\right)
\end{aligned}
$$

as can be easily demonstrated as above. These two relations (10) and (11) show that $\xi_{1}$ and $\xi_{2}$ are congruent respectively to $(k-1)$ and 0 modulo $k$.

(R5): For $k=s^{\prime 2}-2$ with $s^{\prime}$ odd, the rank $r=2$ [11], and the only two sets of solutions are

$$
\begin{aligned}
& \left(t_{1}, \xi_{1}\right)=\left(\frac{1}{2}\left(s^{\prime}-2\right)\left(s^{\prime}+1\right), \frac{1}{2}\left(s^{\prime 2}-2\right)\left(s^{\prime}-1\right)-1\right) \\
& \left(t_{2}, \xi_{2}\right)=\left(\frac{s^{\prime}}{2}\left(s^{\prime}+1\right)-1, \frac{1}{2}\left(s^{\prime 2}-2\right)\left(s^{\prime}+1\right)\right)
\end{aligned}
$$


Table 3. Combination of parameters $m$ and $v$ for $2 \leq n \leq 12$

\begin{tabular}{|c|c|c|c|c|c|c|c|c|c|c|c|c|}
\hline \multirow[t]{2}{*}{$m$} & & \multicolumn{11}{|c|}{$v$} \\
\hline & & 1 & 2 & 3 & 4 & 5 & 6 & 7 & 8 & 9 & 10 & 11 \\
\hline \multirow{11}{*}{$n$} & 2 & 1_ & & & & & & & & & & \\
\hline & 3 & 1 & 1 & & & & & & & & & \\
\hline & 4 & 1 & 1 & 1 & & & & & & & & \\
\hline & 5 & $1_{-}$ & 2 & 2 & 1 & & & & & & & \\
\hline & 6 & 1 & I & T & / & 1 & & & & & & \\
\hline & 7 & $1_{-}$ & 3 & 2 & 2 & 3 & 1 & & & & & \\
\hline & 8 & 1 & I & 3 & I & 3 & / & 1 & & & & \\
\hline & 9 & 1 & 4 & I & 2 & 2 & / & 4 & 1 & & & \\
\hline & 10 & 1 & l & 3 & / & 5 & 1 & 3 & / & 1 & & \\
\hline & 11 & $1_{-}$ & 5 & 4 & 3 & 2 & 2 & 3 & 4 & 5 & 1 & \\
\hline & 12 & $1_{-}$ & / & I & l & 3 & I & 4 & / & I & / & 1 \\
\hline
\end{tabular}

as can easily be shown as above. These two relations (12) and (13) show that $\xi_{1}$ and $\xi_{2}$ are congruent respectively to $(k-1)$ and 0 modulo $k$.

There are other cases of interest as shown in the next two Propositions:

Proposition 3. For $\forall n \in \mathbb{Z}^{+}, \exists k, \xi, \mu<k, i, j \in \mathbb{Z}^{+}, k$ non-square, such that if $\xi_{i}$ are solutions of (1) with $\xi_{i} \equiv \mu_{j}(\bmod k)$, and $(R 6)$ if $k$ is twice a triangular number $k=n(n+1)=2 T_{n}$, then the set of $\mu_{j}$ includes $\left\{0, n,\left(n^{2}-1\right),(k-1)\right\}$, with $1 \leq j \leq v$.

Proof. Let $n, k, \xi, \mu<k, i, j \in \mathbb{Z}^{+}, k$ non-square, and $\xi_{i}$ solutions of (1). Let $\xi_{i} \equiv \mu_{j}(\bmod k)$ with $1 \leq j \leq v$. As the ratio $\xi_{i}\left(\xi_{i}+1\right) / k$ must be integer, $\xi_{i}\left(\xi_{i}+1\right) \equiv 0(\bmod k)$ or $\mu_{j}\left(\mu_{j}+1\right) \equiv 0(\bmod n(n+1))$ which is obviously satisfied if $\mu_{j}=n$ or $\mu_{j}=\left(n^{2}-1\right)$.

Finally, this last proposition gives a general expression of the congruence $\xi_{i}(\bmod k)$ for most cases to find the remainders $\mu_{j}$ other than 0 and $(k-1)$.

Proposition 4. For $\forall n>1 \in \mathbb{Z}^{+}, \exists k, f, \xi, v<n<k, \mu<k, m<n, i, j \in \mathbb{Z}^{+}, k$ non-square, let $\xi_{i}$ be solutions of (1) with $\xi_{i} \equiv \mu_{j}(\bmod k)$, let $f$ be a factor of $k$ such that $f=k / n$ with $f \equiv v(\bmod n)$ and $k \equiv v n\left(\bmod n^{2}\right)$, then the set of $\mu_{j}$ includes either $\{0, m f,((n-m) f-1),(k-1)\}$ or $\{0,(m f-1),(n-m) f,(k-1)\}$, where $m$ is an integer multiplier of $f$ in the congruence relation and such that $m<n / 2$ or $m<(n+1) / 2$ for $n$ being even or odd respectively, and $1 \leq j \leq v$.

Proof. Let $n>1, k, f, \xi, \mu<k, m<n, i, j<n<k \in \mathbb{Z}^{+}, k$ non-square, and $\xi_{i}$ a solution of (1). Let $\xi_{i} \equiv$ $\mu_{j}(\bmod k)$ with $1 \leq j \leq v$. As the ratio $\xi_{i}\left(\xi_{i}+1\right) / k$ must be integer, $\xi_{i}\left(\xi_{i}+1\right) \equiv 0(\bmod k)$ or $\mu_{j}\left(\mu_{k}+1\right) \equiv$ $0(\bmod f n)$. For a proper choice of the factor $f$ of $k$, let $\mu_{j}$ be a multiple of $f, \mu_{j}=m f$, then $m(m f+1) \equiv$ $0(\bmod n)$. As $f \equiv v(\bmod n)$, one has

$$
m(m v+1) \equiv 0(\bmod n) .
$$

Let now $\left(\mu_{j}+1\right)$ be a multiple of $f, \mu_{j}+1=m f$, then $m(m f-1) \equiv 0(\bmod n)$ or

$$
m(m v-1) \equiv 0(\bmod n) \text {. }
$$

An appropriate combination of integer parameters $m$ and $v$ guarantees that (14) and (15) are satisfied. Proposition 1 yields the other remainder value as $m f+(n-m) f-1=k-1$ and $(m f-1)+(n-m) f=$ $k-1$.

The appropriate combinations of integer parameters $m$ and $v$ are given in Table 3 for $2 \leq n \leq 12$. The sign - in subscript corresponds to the remainder $(m f-1)$; the sign / indicates an absence of combination.

One deduces from Table 3 the following simple rules:

1) $\forall n \in \mathbb{Z}^{+}$, only those values of $v$ that are co-prime with $n$ must be kept, all other combinations (indicated by / in Table 3) must be discarded as they correspond to combinations with smaller values of $n$ and $v$; 
for $n$ even, this means that all even values of $v$ must be discarded. For example, $v=2$ and $n=4$ are not co-prime and their combination corresponds to $v=1$ and $n=2$.

2) For $v=1$ and $v=n-1$, all values of $m$ are $m=1$ with respectively the remainders $(m f-1)$ and $m f$.

3) For $\forall n, i \in \mathbb{Z}^{+}, n$ odd, $2 \leq i \leq(n-1) / 2$, and for $v=(n-(2 i-3)) / 2$ and $v=(n+(2 i-3)) / 2$, all the values of $m$ are $m=i$.

4) For $\forall n \in \mathbb{Z}^{+}, n$ odd, and for $v=2$ and $v=n-2$, the remainders are respectively $m f$ and $(m f-1)$.

5) For $\forall n, i \in \mathbb{Z}^{+}, n$ even, $2 \leq i \leq n / 2$, and for $v=(n-(2 i-3)) / 2$ and $v=(n+(2 i-3)) / 2$, all the values of $m$ are $m=i$.

Expressions of $\mu_{i}$ are given in Table 4 for $2 \leq n \leq 12$ (with codes Env). For example, for $k \equiv 12 v\left(\bmod 12^{2}\right)$ and $v=5$ (code E125), i.e. $k=60,204,348, \ldots, \xi_{i} \equiv \mu_{j}(\bmod k)$ with the set of remainders $\mu_{j}$ including $\{0, m f,((n-m) f-1),(k-1)\}$ with $m=3$ (see Table 3$)$ and $f=k / 12=5,17,29$...respectively.

Table 4. Expressions of $\mu_{j}$ for $2 \leq n \leq 12$

\begin{tabular}{|c|c|c|c|c|c|c|}
\hline$n$ & $v$ & $m$ & $k \equiv$ & $f$ & $\mu_{j}$ & Code \\
\hline 2 & 1 & 1 & $2(\bmod 4)$ & $k / 2$ & $0,(k / 2)-1, k / 2, k-1$ & E21 \\
\hline \multirow[t]{2}{*}{3} & 1 & 1 & $3(\bmod 9)$ & \multirow[t]{2}{*}{$k / 3$} & $0,(k / 3)-1,2 k / 3, k-1$ & E31 \\
\hline & 2 & 1 & $6(\bmod 9)$ & & $0, k / 3,(2 k / 3)-1, k-1$ & E32 \\
\hline \multirow[t]{2}{*}{4} & 1 & 1 & $4(\bmod 16)$ & \multirow[t]{2}{*}{$k / 4$} & $0,(k / 4)-1,3 k / 4, k-1$ & E41 \\
\hline & 3 & 1 & $12(\bmod 16)$ & & $0, k / 4,(3 k / 4)-1, k-1$ & E43 \\
\hline \multirow[t]{4}{*}{5} & 1 & 1 & $5(\bmod 25)$ & \multirow[t]{4}{*}{$k / 5$} & $0,(k / 5)-1,4 k / 5, k-1$ & E51 \\
\hline & 2 & 2 & $10(\bmod 25)$ & & $0,2 k / 5,(3 k / 5)-1, k-1$ & E52 \\
\hline & 3 & 2 & $15(\bmod 25)$ & & $0,(2 k / 5)-1,3 k / 5, k-1$ & E53 \\
\hline & 4 & 1 & $20(\bmod 25)$ & & $0, k / 5,(4 k / 5)-1, k-1$ & E54 \\
\hline \multirow[t]{2}{*}{6} & 1 & 1 & $6(\bmod 36)$ & \multirow[t]{2}{*}{$k / 6$} & $0,(k / 6)-1,5 k / 6, k-1$ & E61 \\
\hline & 5 & 1 & $30(\bmod 36)$ & & $0, k / 6,(5 k / 6)-1, k-1$ & E65 \\
\hline \multirow[t]{6}{*}{7} & 1 & 1 & $7(\bmod 49)$ & \multirow[t]{6}{*}{$k / 7$} & $0,(k / 7)-1,6 k / 7, k-1$ & E71 \\
\hline & 2 & 2 & $14(\bmod 49)$ & & $0,3 k / 7,(4 k / 7)-1, k-1$ & E72 \\
\hline & 3 & 3 & $21(\bmod 49)$ & & $0,2 k / 7,(5 k / 7)-1, k-1$ & E73 \\
\hline & 4 & 3 & $28(\bmod 49)$ & & $0,(2 k / 7)-1,5 k / 7, k-1$ & E74 \\
\hline & 5 & 2 & $35(\bmod 49)$ & & $0,(3 k / 7)-1,4 k / 7, k-1$ & E75 \\
\hline & 6 & 1 & $42(\bmod 49)$ & & $0, k / 7,(6 k / 7)-1, k-1$ & E76 \\
\hline \multirow[t]{4}{*}{8} & 1 & 1 & $8(\bmod 64)$ & \multirow[t]{4}{*}{$k / 8$} & $0,(k / 8)-1,7 k / 8, k-1$ & E81 \\
\hline & 3 & 3 & $24(\bmod 64)$ & & $0,(3 k / 8)-1,5 k / 8, k-1$ & E83 \\
\hline & 5 & 3 & $40(\bmod 64)$ & & $0,3 k / 8,(5 k / 8)-1, k-1$ & E85 \\
\hline & 7 & 1 & $56(\bmod 64)$ & & $0, k / 8,(7 k / 8)-1, k-1$ & E87 \\
\hline \multirow[t]{6}{*}{9} & 1 & 1 & $9(\bmod 81)$ & \multirow[t]{6}{*}{$k / 9$} & $0,(k / 9)-1,8 k / 9, k-1$ & E91 \\
\hline & 2 & 4 & $18(\bmod 81)$ & & $0,4 k / 9,(5 k / 9)-1, k-1$ & E92 \\
\hline & 4 & 2 & $36(\bmod 81)$ & & $0,2 k / 9,(7 k / 9)-1, k-1$ & E94 \\
\hline & 5 & 2 & $45(\bmod 81)$ & & $0,(2 k / 9)-1,7 k / 9, k-1$ & E95 \\
\hline & 7 & 4 & $63(\bmod 81)$ & & $0,(4 k / 9)-1,5 k / 9, k-1$ & E97 \\
\hline & 8 & 1 & $72(\bmod 81)$ & & $0, k / 9,(8 k / 9)-1, k-1$ & E98 \\
\hline \multirow[t]{4}{*}{10} & 1 & 1 & $10(\bmod 100)$ & \multirow[t]{4}{*}{$k / 10$} & $0,(k / 10)-1,9 k / 10, k-1$ & E101 \\
\hline & 3 & 3 & $30(\bmod 100)$ & & $0,3 k / 10,(7 k / 10)-1, k-1$ & E103 \\
\hline & 7 & 3 & $70(\bmod 100)$ & & $0,(3 k / 10)-1,7 k / 10, k-1$ & E107 \\
\hline & 9 & 1 & $90(\bmod 100)$ & & $0, k / 10,(9 k / 10)-1, k-1$ & E109 \\
\hline \multirow[t]{10}{*}{11} & 1 & 1 & $11(\bmod 121)$ & \multirow[t]{10}{*}{$k / 11$} & $0,(k / 11)-1,10 k / 11, k-1$ & E111 \\
\hline & 2 & 5 & $22(\bmod 121)$ & & $0,5 k / 11,(6 k / 11)-1, k-1$ & E112 \\
\hline & 3 & 4 & $33(\bmod 121)$ & & $0,(4 k / 11)-1,7 k / 11, k-1$ & E113 \\
\hline & 4 & 3 & $44(\bmod 121)$ & & $0,(3 k / 11)-1,8 k / 11, k-1$ & E114 \\
\hline & 5 & 2 & $55(\bmod 121)$ & & $0,2 k / 11,(9 k / 11)-1, k-1$ & E115 \\
\hline & 6 & 2 & $66(\bmod 121)$ & & $0,(2 k / 11)-1,9 k / 11, k-1$ & E116 \\
\hline & 7 & 3 & $77(\bmod 121)$ & & $0,3 k / 11,(8 k / 11)-1, k-1$ & E117 \\
\hline & 8 & 4 & $88(\bmod 121)$ & & $0,4 k / 11,(7 k / 11)-1, k-1$ & E118 \\
\hline & 9 & 5 & $99(\bmod 121)$ & & $0,(5 k / 11)-1,6 k / 11, k-1$ & E119 \\
\hline & 10 & 1 & $110(\bmod 121)$ & & $0, k / 11,(10 k / 11)-1, k-1$ & E1110 \\
\hline \multirow[t]{4}{*}{12} & 1 & 1 & $12(\bmod 144)$ & \multirow[t]{4}{*}{$k / 12$} & $0,(k / 12)-1,11 k / 12, k-1$ & E121 \\
\hline & 5 & 3 & $60(\bmod 144)$ & & $0,3 k / 12,(9 k / 12)-1, k-1$ & E125 \\
\hline & 7 & 4 & $84(\bmod 144)$ & & $0,(4 k / 12)-1,8 k / 12, k-1$ & E127 \\
\hline & 11 & 1 & $132(\bmod 144)$ & & $0, k / 12,(11 k / 12)-1, k-1$ & E1211 \\
\hline
\end{tabular}


Table 5. Values of $\mu_{j}$ for $2 \leq k \leq 120$

\begin{tabular}{|c|c|c|c|c|c|}
\hline$k$ & $\mu_{j}$ & References & $k$ & $\mu_{j}$ & References \\
\hline 2 & 0,1 & R1,R6,E21 & 63 & $0,27,35,62$ & E72,E97 \\
\hline 3 & 0,2 & R1,E31 & 65 & 0,64 & R3 \\
\hline 5 & 0,4 & R1,R3,E51 & 66 & $0,11,21,32,33,44,54,65$ & $\mathrm{E} 21+\mathrm{E} 31+\mathrm{E} 65+\mathrm{E} 116$ \\
\hline 6 & $0,2,3,5$ & R6,E21,E32,E61 & 67 & 0,66 & $\mathrm{R} 1$ \\
\hline 7 & 0,6 & R1,R5,E71 & 68 & $0,16,51,67$ & E41 \\
\hline 8 & 0,7 & R2,R4,E81 & 69 & $0,23,45,68$ & E32 \\
\hline 10 & $0,4,5,9$ & E21,E52,E101 & 70 & $0,14,20,34,35,49,55,69$ & E21+E54+E73+E107 \\
\hline 11 & 0,10 & R1,E111 & 71 & 0,70 & $\mathrm{R} 1$ \\
\hline 12 & $0,3,8,11$ & R6,E31,E43,E121 & 72 & $0,8,63,71$ & R6,E81,E98 \\
\hline 13 & 0,12 & R1 & 73 & 0,72 & R1 \\
\hline 14 & $0,6,7,13$ & E21,E72 & 74 & 0,73 & $?$ \\
\hline 15 & $0,5,9,14$ & E32,E53 & 75 & $0,24,50,74$ & E31 \\
\hline 17 & 0,16 & R1,R3 & 76 & $0,19,56,75$ & E43 \\
\hline 18 & $0,8,9,17$ & E21,E92 & 77 & $0,21,55,76$ & E74,E117 \\
\hline 19 & 0,18 & $\mathrm{R} 1$ & 78 & $0,12,26,38,39,51,65,77$ & $\mathrm{E} 21+\mathrm{E} 32+\mathrm{E} 61$ \\
\hline 20 & $0,4,15,19$ & R6,E41,E54 & 79 & 0,78 & R1,R5 \\
\hline 21 & $0,6,14,20$ & E31,E73 & 80 & 0,79 & $\mathrm{R} 4$ \\
\hline 22 & $0,10,11,21$ & E21,E112 & 82 & $0,40,41,81$ & E21 \\
\hline 23 & 0,22 & R1,R5 & 83 & 0,82 & R1 \\
\hline 24 & 0,23 & $\mathrm{R} 4$ & 84 & $0,27,56,83$ & E31,E127 \\
\hline 26 & $0,12,13,25$ & E21 & 85 & $0,34,50,84$ & E52 \\
\hline 27 & 0,26 & R2 & 86 & $0,42,43,85$ & E21 \\
\hline 28 & $0,7,20,27$ & E43,E74 & 87 & $0,29,57,86$ & E32 \\
\hline 29 & 0,28 & R1 & 88 & $0,32,55,87$ & E83,E118 \\
\hline 30 & $0,5,24,29$ & R6,E51,E65 & 89 & 0,88 & $\mathrm{R} 1$ \\
\hline 31 & 0,30 & R1 & 90 & $0,9,80,89$ & R6,E91,E109 \\
\hline 32 & 0,31 & R2 & 91 & $0,13,77,90$ & E75 \\
\hline 33 & $0,11,21,32$ & E32,E113 & 92 & $0,23,68,91$ & E43 \\
\hline 34 & $0,16,17,33$ & E21 & 93 & $0,30,62,92$ & E31 \\
\hline 35 & $0,14,20,34$ & E52,E75 & 94 & $0,46,47,93$ & E21 \\
\hline 37 & 0,36 & R1,R3 & 95 & $0,19,75,94$ & E54 \\
\hline 38 & $0,18,19,37$ & E21 & 96 & $0,32,63,95$ & E32 \\
\hline 39 & $0,12,26,38$ & E31 & 97 & 0,96 & R1 \\
\hline 40 & $0,15,24,39$ & E53,E85 & 98 & $0,48,49,97$ & E21 \\
\hline 41 & 0,40 & R1 & 99 & $0,44,54,98$ & E92,E119 \\
\hline 42 & $0,6,35,41$ & R6,E61,E76 & 101 & 0,100 & R1,R3 \\
\hline 43 & 0,42 & $\mathrm{R} 1$ & 102 & $0,50,51,102$ & E21 \\
\hline 44 & $0,11,32,43$ & E43,E114 & 103 & 0,102 & R1 \\
\hline 45 & $0,9,35,44$ & E54,E95 & 104 & 0,103 & $?$ \\
\hline 46 & $0,22,23,245$ & E21 & 105 & $0,14,20,35,69,84,90,104$ & E32+E51+E71 \\
\hline 47 & 0,46 & R1,R5 & 106 & $0,52,53,105$ & E21 \\
\hline 48 & 0,47 & R4 & 107 & 0,106 & R1 \\
\hline 50 & $0,24,25,49$ & E21 & 108 & $0,27,80,107$ & $\mathrm{E} 43$ \\
\hline 51 & $0,17,33,50$ & E32 & 109 & 0,108 & R1 \\
\hline 52 & $0,12,39,51$ & E41 & 110 & $0,10,99,109$ & R6,E101,E1110 \\
\hline 53 & 0,52 & R1 & 111 & $0,36,74,110$ & E31 \\
\hline 54 & $0,26,27,53$ & E21 & 112 & $0,48,63,111$ & E72 \\
\hline 55 & $0,10,44,54$ & E51,E115 & 113 & 0,112 & R1 \\
\hline 56 & $0,7,48,55$ & R6,E71,E87 & 114 & $0,56,57,113$ & E21 \\
\hline 57 & $0,18,38,56$ & E31 & 115 & $0,45,69,114$ & E53 \\
\hline 58 & $0,28,29,57$ & E21 & 116 & $0,28,87,115$ & E41 \\
\hline 59 & 0,58 & R1 & 117 & $0,26,90,116$ & E94 \\
\hline 60 & $0,15,44,59$ & E43,E125 & 118 & $0,58,59,117$ & E21 \\
\hline 61 & 0,60 & R1 & 119 & 0,118 & R1,R5 \\
\hline 62 & $0,30,31,61$ & E21 & 120 & $0,15,104,119$ & E87 \\
\hline
\end{tabular}


Values of the remainders $\mu_{j}$ are given in Table 5 for $2 \leq k \leq 120$, with rule (R) and expression (E) codes as references. $\mathrm{R}$ and $\mathrm{E}$ codes separated by commas imply that all references apply simultaneously to the case; E codes separated by + mean that all expressions apply to the case; some expression references are sometimes missing. One observes that in two cases (for $k=74$ and 104), expressions could not be found (indicated by question marks).

Table 5 gives correctly the values of the remainder pairs in most of the cases. There are although some exceptions and some values missing.

Among the exceptions to the values given in Table 5 , for $n=2$, remainders values for $k=$ $30,42,74,90,110, \ldots$ are different from the theoretical ones in Table 4 . Furthermore, for $k=66,70,78,105, \ldots$, additional remainders exist. Expressions are missing for $k=74$ (E21) and 104 (E85). Finally, one observes also that for 16 cases, some Rules or Expressions supersede some other Expressions (indicated by Ra > Exy or Exy $>$ Ezt), as reported in Table 6. For example, Rule 6 supersedes Expression 21 (R6 > E21) for $k=30,42,90,110$, i.e., $k=2 T_{5}, 2 T_{6}, 2 T_{9}, 2 T_{10}, \ldots$ and more generally for all $k=2 T_{i}$ for $i \equiv 1,2(\bmod 4)$.

Table 6. Rules and Expressions superseding other Rules and Expressions

\begin{tabular}{c|l}
$k$ & \\
\hline 24 & R4 $>$ E32; R4 > E83 \\
\hline 30 & R6 > E21; R6 > E31; R6 > E103; E51 > E103; E65 > E103 \\
\hline 42 & R6 > E21; R6 > E32 \\
\hline 48 & R4 > E31 \\
\hline 56 & R6 > E43 \\
\hline 60 & E43 > E32; E43 > E52 \\
\hline 65 & R3 > E53 \\
\hline 72 & R6 > E43 \\
\hline 80 & R4 > E51 \\
\hline 84 & E31 $>$ E41; E31 > E75 \\
\hline 90 & R6 > E21; R6 > E53 \\
\hline 102 & E21 > E31; E21 > E65 \\
\hline 110 & R6 > E21; R6 > E52 \\
\hline 114 & E21 > E32; E21 > E61 \\
\hline 119 & R1 $>$ E73; R5 > E73 \\
\hline 120 & E87 > R4; E87 > E31; E87 > E54
\end{tabular}

Note that 11 of these 16 values of $k$ are multiples of 6 , the others are $2 \bmod 6$ and $5 \bmod 6$ for, respectively three and two cases. One notices as well, that generally, Ra and Exy supersede Ezt with $x<z$ and $t<y$, except for $k=60$ and 120 .

\section{Conclusions}

We have shown that, for indices $\xi$ of triangular numbers multiples of other triangular numbers, the remainders in the congruence relations of $\xi$ modulo $k$ always come in pairs whose sum always equal $(k-1)$, always include 0 and $(k-1)$, and only 0 and $(k-1)$ if $k$ is prime, or an odd power of a prime, or an even square plus one or an odd square minus one or minus two. If the multiplier $k$ is twice a triangular number of $n$, the set of remainders includes also $n$ and $\left(n^{2}-1\right)$ and if $k$ has integer factors, the set of remainders include multiple of a factor following certain rules. Finally, algebraic expressions are found for remainders in function of $k$ and its factors. Several exceptions are noticed as well as reported above and it appears that there are superseding rules between the various rules and expressions.

This approach allows eliminating in numerical searches those $(k-v)$ values of $\xi_{i}$ that are known not to provide solutions of (1), where $v$ is the even number of remainders. The gain is typically in the order of $k / v$, with $v \ll k$ for large values of $k$.

Conflicts of Interest: The author declares no conflict of interest.

Data Availability: Data required for this research is included within this paper.

Funding Information: The author received no financial support for the research authorship and / or publication of this article.

\section{References}


[1] Andrews, G. E. (1971). Number Theory. Dover Publications, New York.

[2] Weisstein, E. W. (2021). Triangular Number, in MathWorld-A Wolfram Web Resource. Last accessed 14 February 2021. http:/ / mathworld.wolfram.com/TriangularNumber.html.

[3] Cunningham, A. (1901). Mathematical Questions and Solutions in Continuation of the Mathematical Columns of "the Educational Times", Volume 75, F. Hodgson, 87-88.

[4] de Joncourt, E. (1762). The Nature and Notable Use of the Most Simple Trigonal Numbers. The Hague: Husson.

[5] Roegel, D. (2013). A reconstruction of Joncourt's table of triangular numbers (1762). LOCOMAT project. Last accessed 14 February 2021. https:/ /locomat.loria.fr/joncourt1762/joncourt1762doc.pdf.

[6] Dickson, L. E. (2005). History of the Theory of Numbers, Vol. II: Diophantine Analysis. Dover Publications, New York, p. 587.

[7] Chahal, J. S., \& D'Souza, H. (1993). Some remarks on Triangular Numbers, in Number Theory with an Emphasis on the Markoff Spectrum, A. D. Pollington and W. Mran, eds., Marcel Dekker Inc., New York.

[8] Breiteig, T. (2015). Quotients of triangular numbers. The Mathematical Gazette, 99(545), 243-255.

[9] Pletser, V. (2021). Recurrent relations for multiple of triangular numbers being triangular numbers. ArXiv 2101.00998. Last accessed 14 February 2021. http:/ / arxiv.org/abs/2101.00998.

[10] Sloane, N. J. A., (2018). The On-Line Encyclopedia of Integer Sequences. Last accessed 14 February 2021. Published electronically at https:/ / oeis.org.

[11] Pletser V. (2021). Searching for multiple of triangular numbers being triangular numbers. Research Gate, DOI: 10.13140/RG.2.2.35428.91527. https://www.researchgate.net/publication/349788977 .

( 2021 by the authors; licensee PSRP, Lahore, Pakistan. This article is an open access article distributed under the terms and conditions of the Creative Commons Attribution (CC-BY) license (http://creativecommons.org/licenses/by/4.0/). 